\title{
Ueber Nabelschnurbruch und Blasenbauchspalte mit Cloakenbildung von Seiten des Dïnndarmes.
}

Von

\author{
Dr. v. Steinbüchel, \\ emerit. Assistent an der Klinik und Privatdocent für Geburtshilfe nnd Gynäkologie an der \\ Universität Graz. \\ (Mit 8 Abbildungen im Text.)
}

Am 5. September 1894 brachte eine Hebamme ein neugeborenes Kind an die hiesige Frauenklinik mit der Bitte; "man möchte ihr sagen, ob das Kind männlichen oder weiblichen Geschlechtes sei".

Ueber die Mutter des Kindes und den Verlauf der Geburt machte sie folgende Angaben: Frau S. H., 27 Jahre alt, Primipara, angeblich immer gesund, gebar am 4. September 1894 um $3 / 43$ Uhr Nachmittags spontan ein reifes, lebendes Kind. Die Geburt war am normalen Ende der Gravidität in I. Schädellage erfolgt. Placenta und Eihäute wurden $1 / 2$ Stunde nach Austreibung des Kindes spontan geboren. Letztere zeigten keinerlei Abnormitäten.

In der Klinik wurde folgender Befund aufgenommen: Kind reif, kräftig entwickelt, männlichen Geschlechts, $52 \mathrm{~cm}$ lang, $3260 \mathrm{~g}$ schwer. Nabel- und Unterbauchgegend in mehrfacher Beziehung abnorm (vergl. die Abbildung).

Etwas mehr der Symphyse genähert, als es gewöhnlich der Fall ist, geht die sulzreiche Nabelschnur ab.

Ihr Anfangsstück ist zu einer etwa nussgrossen querovalen Geschwulst (Nabelschnurhernie) umgewandelt, deren querer Durchmesser $3 \frac{1}{2} \mathrm{~cm}$, deren Höhe $2 \% \mathrm{~cm}$ beträgt, so dass die Nabelschnur scheinbar von der Kuppe des Tumors entspringt. 
Figur 1.

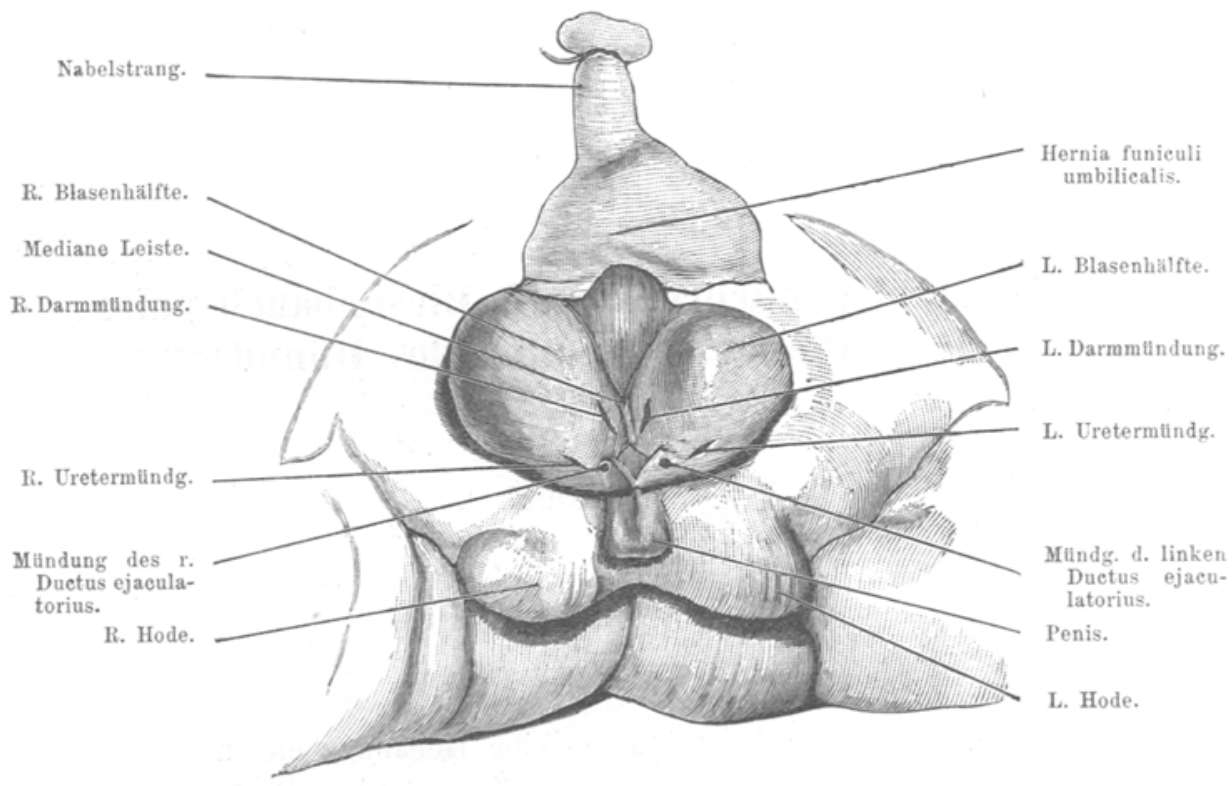

Die Bruchhülle wird am Rande von einem sehr schmalen Saum verdünnter Bauchhaut, im Uebrigen vom Amnion gebildet.

Nach unten zu geht der Bruchsack in eine pralle, dunkelrothe, sammetartig glänzende Geschwulst über, die sich beim Schreien des Kindes vergrössert und bei Berührung leicht blutet, die ektropionirte innere Blasenwand. Dieselbe ist durch eine von oben nach unten verlaufende seichte Furche in zwei Hälften getheilt. Paarig zu beiden Seiten der Furche liegen die schlitzförmigen Ureterenmündungen $(u)$ und unter diesen die knopfartig vorspringenden Oeffnungen der Ductus ejaculatorii (e). Etwas nach oben und innen von den Ureterenostien, welche sich durch den stossweise hervorquellenden Harn sofort als solehe kenntlich machten, finden sich zwei längliche spaltförmige Oeffnungen, aus welchen sich Meconium entleert.

Zwischen denselben springt das Gewebe leistenartig vor. Diese mediane Leiste theilt sich nach oben sowohl als nach unten zu in zwei Schenkel, welche in die Umgebung flach auslaufen. - Der am unteren Ende der ektropionirten Blase befindliche Penis ist sehr klein, zeigt jedoch eine Andeutung der 
Fichelbildung und ein kurzes Praeputium. Die Urethra ist offen und stellt sich in ihrem ganzen Verlaufe als eine an der Oberfläche des Penis verlaufende seichte Rinne dar.

Das Scrotum ist ziemlich gut ausgebildet und durch eine mediane Furche in zwei sackartige Hälften getheilt. Die Hoden liegen beiderseits bereits ausserhalb der Bauchhöhle, knapp vor den äusseren Pforten der Leistenkanäle.

Der Anus ist nur als seichtes Grübchen andeutungsweise vorhanden. - Die Symphysenenden sind $5 \mathrm{~cm}$ von einander entfernt.

Auf Wunsch der Eltern wird am 5. Sept. 94 die operative Beseitigung der Nabelhernie vorgenommen. Narcose, Umschneidung des Bruchsackes, Versuch, das durch die Anfrischung abgetrennte circuläre Stück Cutis, sowie das ziemlich dicke Amnion des Bruchsackes rom Peritoneum abzupräpariren.

Unter dem Amnion finden sich Reste von Nabelstrangsulze. Die Bruchpforte war weit, den Inhalt des Bruches bildeten Dünndarmschlingen. - Durchtrennung der Nabelgefässe und Einzelligatur derselben. - Die Umbilicalvene verlief im oberen Antheile der elliptischen Wunde, die beiden Arterien unten zu beiden Seiten der Mittellinie.

Beim Versuch das Amnion abzulösen, reisst das Peritoneum entsprechend der Kuppe des Bruchsackes ein, und Darmschlingen werden herausgepresst, deren Reposition jedoch ziemlich leicht gelingt. Unter Leitung des in die Bauchhöhle eingeführten Fingers werden 4 durchgreifende Bauchdeckennähte angelegt, der Riss im Peritoneum durch feine Seidennähte geschlossen und nun unter ziemlicher Spannung die Bauchdeckensuturen geknotet. Die Nahtlinie wird mit Dermatol bestreut, mit steriler Gaze bedeckt und der Verband durch eirculäre Heftpflasterstreifen befestigt. Das Kind wird einer Wöchnerin an die Brust gelegt; weil es jedoch nicht saugt, wird ihm künstlich mittelst Jüffels die vorher aus der Brust gedrückte Muttermileh eingeflösst und zwar bis zum nächsten Morgen ca. 6 Esslöffel voll.

6. Sept. Das Kind ist ruhig, schläft gut, Temp. afebril, aus der Blasendarmfistel entleert sich Meconium in äusserst geringer Menge. Heute nimmt das Kind die Brust, trinkt kräftig, schläft ruhig, Temp. afebril, kein Abgang von Meconium.

7. Sept. Status idem, nur ist der Bauch etwas aufgetrieben, auf Druck entleert sich aus der Fistelöffnung spärlicher Darm- 
inhalt, der nicht mehr ganz die Eigenschaften des Meconiums aufweist, sondern schon Beimengung von Milchkoth erkennen lässt.

Der bestehenden Kothstauung halber muss operativ für freieren Abfluss des Darminhaltes gesorgt werden. Es wurden die beiden Darmfistelöffnungen sondirt. Von beiden Oeffnungen gelangt man nach unten gegen den Anus hin in einen Blindsack, andererseits nach oben und links hin weit in ein Darmstück, das die beiden Fisteln trennende Septum reicht nur wenige Millimeter nach innen, dann berühren sich schon zwei eingeführte Sonden. Schaffung einer weiten Tarmöffnung durch Spaltung dieses Septums erschien durch die Gefahr abnormer Gährungsvorgänge in dem gegen den Anus hin sich erstreckenden Blindsacke contraindicirt, es blieb daher nur die Anlegung eines künstlichen Afters an der durch das Grübchen angedeuteten Stelle übrig. Dies wurde nun in der Art ausgeführt, dass von einer der Mündungen des Anus vesicalis aus mittelst einer dicken, ziemlich stark gekrümmten Knopfsonde das blinde Darmende gegen das Perineum gedrängt wurde, bis man vom Damm aus den Sondenknopf fühlen konnte. Präparirend wird die jetzt noch dazwischen liegende etwa $1 / 2 \mathrm{~cm}$ dicke Gewebsschicht durchtrennt. Während des vorsichtigen Tieferdringens liess sich ziemlich deutlich ein vom Analgrübchen zum Darmblindsack ziehender Bindegewebsstrang erkennen, welcher von dem Sphincter ani externus eng umschlossen wurde. Endlich erscheint das vom Sondenknopf vorgedrängte Darmende in der Tiefe der Wunde. Nach Eröffnung desselben wird die Darmschleimhaut ringsum mit der Haut durch Seidenknopfnähte vereinigt und die Nahtlinie mit Dermatolpulver bedeckt. Aus der neu angelegten Darmöffnung entleeren sich nunmehr gelbliche Fäces von normaler Consistenz.

Der weitere Verlaul war ungestört, so lange das Kind in der Klinik beobachtet werden konnte. Der Bauchverband (sterile Gaze durch Heftpflasterstreifen fixirt) wurde, so oft er durch Harn oder Fäces verunreinigt war, erneuert und die reactionslos aussehende Nahtlinie mit Dermatolpulver bestreut. Ein mit Borsalbe bestrichenes Gazeläppchen deckt die sehr leicht blutende Blasenschleimhaut. Das Kind trinkt jetzt gut an der Ammenbrust, schreit kräftig und schläft normal. Stuhl von normaler Qualität und Quantität.

Aeusserer Verhältnisse halber musste das Kind an 9. Sept., also vier Tage nach Operation der Nabelhernie, den Eltern über- 
geben und der in diesem Falle schon voraussichtlich mangelhaften häuslichen Pflege überlassen werden.

Von da ab soll das Kind keinen Stuhl mehr gehabt haben und das Abdomen stets zunehmend stärker aufgetrieben und gespannt gewesen sein, weshalb eine Hebamme behufs Verabfolgung eines Klysmas geholt wurde. Dasselbe soll erfolglos geblieben sein.

10. Sept. Unter Symptomen ron Ileus trat angeblich heute Nachmittags der Exitus ein. - Wie sich später zeigte, war beim Einführen des ziemlich dicken Ansatzrohres der „zinnernen Klystierspritze" das an die Haut genähte Darmstück abgerissen worden, und ergoss sich nun wahrscheinlich zum grössten Theil der Inhalt der Klystierspritze in die freie Bauchhöhle!

Theilweise aus dem Sectionsbefunde (Herr Dr. Brosch, Assistent an dem path.-anat. Institut des Herrn Prof. Dr. Eppinger), theilweise aus dem bei genauerer Besichtigung des Präparates gewonnenen Bilde möchte ich noch Folgendes besonders hervorheben: „Die Unterbauchgegend erscheint verkürzt. An Stelle des im unteren Drittel des Bauches gelegenen Nabels findet sich eine durch Nähte verschlossene sagittale lineare Operationswunde ${ }^{1}$ ).

Eine Besichtigung der Vorderwand der Bauchhöhle von innen her ergiebt folgende Verhältnisse: Die beiden Nieren liegen abnorm tief, die rechte in der Höhe des Promontoriums mit um $45^{\circ}$ gedrehter Längsachse, so dass sie in einer halbqueren Lage der Wirbelsäule anliegt, die linke liegt um die Höhe eines Wirbelkörpers höher und ist im Uebrigen normal gelagert. Die Nieren sind gross, zeigen auffallenderweise keine Randkerbungen und keine Lappung und ist zu erkennen, dass die Ureteren nahezu doppelt so dick sind, als es der Norm entspricht. - In der Medianlinie erscheint ein Stück Darmrohr, das nach ungefähr $1 \mathrm{~cm}$ langem Verlaufe sich in zwei sehr ungleich geartete Schenkel theilt. Der eine Schenkel führt in der Mittellinie parallel dem Verlaufe des Ligamentum vesico-umbilicale medium an die Hinterwand der ektopischen Harnblase, um sich, an deren Hinterseite anlangend, nahezu im rechten Winkel nach vorn umzubiegen und mit paarig gelagerten Oeffnungen, die wir oben beschrieben haben, in diese zu münden. Der andere Schenkel geht unter einem rechten Winkel

1) Die weitere Beschreibung der äusseren Theile deckt sich mit dem früher geschilderten klinischen Befunde. 
von dem eben beschriebenen $a b$ und wendet sich nach hinten und unten, die Richtung gegen die Excavatio des Kreuzbeins einschlagend. Das ersterwähnte unpaarige Rohr, sowie der zur Blase führende Schenkel besitzen annähernd glciches Lumen und beträgt. der Durchmesser dieses Rohres $1 \mathrm{~cm}$. An der Theilungsstelle des unpaaren Rohres ist eine klappenartige Duplicatur der Darmwandung vorhanden, die genau dem anatomischen Bilde der Bauhinschen Klappe entspricht. Das kreuzbeinwärts führende Stück ist reichlich doppelt so dick, als die früher genannten; es stellt einen gut $2 \mathrm{~cm}$ tiefen, an seinem untersten Ende mit einer nabelförmigen: Einziehung versehenen Blindsack dar. Von dieser eingezogenen Stelle führt ein drehrunder Strang in eine Bauchfellduplicatur eingebettet im Bogen nach hinten und entspricht dieser dem am tiefsten Punkte des Coecums entspringenden Wurmfortsatz. - Mehr an der Vorderwand des weiten Blindsackes, der wohl ungezwungen als Coecum aufzufassen ist, inserirt sich ein straff gespannter, gegen den Anus hinziehender solider Gewebsstrang.

$\mathrm{V}$ on den beiden symmetrisch gelegenen, Schlitzform zeigenden Oeffnungen in der Harnblase gelangt man in den ersterwähnten absteigenden Schenkel des Darmrohres, der bis nahezu an die Hinterwand der Blase unpaarig verläuft. Er wird dadurch paarig, dass sich aus der Blasenschleimhaut symmetrisch gelegen rechts und links eine kammartige, ca. $1 \mathrm{~mm}$ hohe Falte erhebt, die im Niveau der schlitzartigen Oeffnungen angelangt, in eine mediane Leiste sich vereinigen. Die Dicke dieses das Darmrohr in zwei Theile sondernden Septums beträgt höchstens $3 \mathrm{~mm}$ und stellt eine das Lumen des Darmrohrs paarig gestaltende Falte dar, welche ca. $3-4 \mathrm{~mm}$ weit in das Darmlumen vorspringt.

Aus dem Darme gelangt man mit der Sonde durch einen operativ geschaffenen künstlichen Anus nach aussen.

Die künstliche Analöffnung communicirt aber durch einen $1 / 2 \mathrm{~cm}$ langen Schlitz zwischen den Nähten mit der von reichlichen Faserstoffmassen erfüllten Bauchhöhle.

Es erübrigt noch auf die hier eingeschlagene Therapie kurz einzugehen.

Karewsky (22) (S. 541) sagt, gestütat auf die Beobachtungen Lindfor's (23) und Kocher's (20) bezüglich der Therapie: "Man wird also die Behandlung so handhaben, dass man kleine, leicht reponirbare Brüche, die gute Bedeckung zu haben scheinen, mit 
einem antiseptischen Verbande bedeckt und nach Abstossung der Nabelschnur erwägt, ob die die Fingeweide bedeckenden Gewebe genug Widerstandsfähigkeit gegen Ruptur besitzen. In allen Fällen, wo Durchbruch droht, oder wo Adhäsionen die Reposition hindern, oder sogar Incarcerationserscheinungen auftreten, ist man zur Radicaloperation berechtigt.

Kleinere Hernien, die mit Sicherheit reponirt werden, können durch percutane Ligatur geheilt werden, da, wo adhärente Darmschlingen - sei es auch nur ein Divertikel - vorliegen, ist die blutige Eröffnung des Bruchsackes indicirt. Man hat zwar wiederholt auch Meckel'sche Divertikel ohne Schaden unterbunden, indessen sollte man die Gefahr der Entstehung einer offenen Nabeldarmfistel und die Möglichkeit des Darmprolapses durch diese nicht unterschätzen."

In unserem Falle wurde von dem exspectativen Verfahren abgesehen, weil es doch eine immerhin recht grosse Menge von Gefahren mit sich bringt, darauf $z u$ bauen, dass sich nach $\Lambda b$ stossung der brandigen Hülle der Defect durch Granulation allmählich schliesse.

Aus diesen Gründen erschien der Entschluss zur Vornahme der Radicaloperation, wenn auch durchaus nicht alle die oben erwähnten Bedingungen erfüllt waren, allein berechtigt.

Es blieb die Wahl zwischen der percutanen Ligatur nach Breus (8) (Reposition des Bruchsackinhaltes - Abklemmung des nunmehr leeren Bruchsackes im Bereiche der Haut an seiner Basis; Abtragung des Bruchsackes und Anlegung der percutanen Ligaturen dicht unter der Klemme) einerseits, der Laparotomie (das heisst Excision des Bruchsackes und Naht der angefrischten Bruchpforte), oder Olshausen's (27) extraperitonealer Methode (Umsehneidung der Bruchpforte durch einen ovalen Schnitt in geringer Entfernung rom Hautrande, Ablösung der Haut und' des Amnios von dem den Bruchsack innen auskleidenden Peritoneum parietale, Unterbindung der durchschnittenen Nabelgefässe, Verschluss der ovalen Wunde der Bauchdecken nach Reposition der Hernie mit tiefen und oberflächlichen Nähten) andererseits.

Die percutane Ligatur verbot sich von selbst durch die Grösse der Bruchöffnung und die Nothwendigkeit, möglichst alles Gewebe der Bauchdecken zu schonen, um die nach der Reposition der Därme immerhin recht beträchtliche Spannung zu vermindern; aber auch mit Rücksicht auf die Gefahr der Darmver- 
letzung wäre dieser Eingriff hier unpassend erschienen. Es blieben daher nur die Radicaloperation mittelst Laparotomie und das Verfahren nach Olshausen übrig, und dieses letztere Verfahren wurde aus den nachfolgenden Gründen gewähIt. Wenn auch nach Lindfors (24 Operationen mit 20 Heilungen), Runge, Klausser, Salmon Larabry ${ }^{\mathbf{1}}$ ) die Resultate der Laparotomie befriedigende zu nennen sind, so stehen denselben doch auch Misserfolge [d'Arcy $\left.\mathrm{P}_{\text {ower }}{ }^{1}\right)$ ] gegenüber; daher muss die 0lshausen'sche Methode als ein grosser Fortschritt in der operativen Behandlung des Nabelschnurbruches für jene Fälle bezeichnet werden, in denen sie eben anwendbar ist.

Sie bietet ja den grossen Vortheil, dass "das Peritoneum erhalten bleibt, was besonders für den leicht möglichen Fall einer Dehiscenz der Wunde die Heilung noch sichern muss, da die vom Peritoneum aus sich bildenden Granulationen die Lücke bald ausfüllen". Allein das Wesentliche der Methode, die Ablösung des Amnions vom Peritoneum ohne Verletzung des letzteren, scheint, ebenso wie in unserem Falle, auch anderen Operateuren nicht immer gelungen zu sein [Dohrn und Runge (31)]. Es ergab sich daher in unserem Falle mit Nothwendigkeit das geschilderte Vorgehen: Versuch nach Olshausen zu operiren; gelingt die Ablösung des Amnion nicht, reisst das Peritoneum ein, dann wird der Bruchsack excidirt, der Bruchinhalt reponirt und die Wundränder der Bauchdecken durch die Naht vereinigt. - Diese Art des therapeutischen Vorgehens dürfte für alle ähnlichen Fälle von Nabelschnurbruch empfehlenswerth sein.

\section{Versuch einer Erklärung.}

So lange man die angeborenen Verschliessungen des Mastdarmes als einfache Hemmungsbildung auffasste, war ein einheitlicher Gesichtspunkt für die Entstehung und Erklärung der so unendlich mannigfaltigen Formen der Atresie nicht zu gewinnen und es war fast selbstverständlich, dass einer jeden der verschiedenen vorkommenden Bildungen ein eigener Entstehungsmodus untergeschoben wurde, der auch oft die Beobachter zu generalisirenden Schlüssen verleitete. Seit der wesentlich der Aufklärung dieser einschneidenden Fragen gewidmeten sachlicher Arbeit von

1) Cit. in Karewsky (22). 
Frank (46) ist es möglich, sämmtliche hier in Betracht kommenden Missbildungen von einem der grössten Verallgemeinerung fähigen Standpunkte zu betrachten.

Frank geht von der Ansicht aus, dass der angeborene Verschluss des Mastdarmes stets durch Verwachsung fötaler Anlagen entsteht.

Frank fasst also die in Rede stehenden Verbildungen nicht als Hemmungsmissbildung, sondern als ein Plus an normalen Vorgängen auf, "als Uebermaass der normaliter bei der Bildung der Ausführungsgänge vor sich gehenden Verwachsungen".

Durch vorgenannte Anschauung brach Frank (1. c.) auf das Entschiedenste mit den entwickelungsgeschichtlich nicht haltbaren Anschauungen, dass alle Nebenmündungen des Mastdarmes als Cloakenreste aufzufassen seien. Endlich trennt er scharf innere und äussere Nebenausmündungen und sucht für letztere die Erklärung aus der Spalte zwischen den paarigen ektodermalen Anlagen, während er erstere als Cloakenreste gelten lässt. - Während sich aber die Arbeit Frank's (l. c.) grösstentheils mit den Nebenausmündungen des Mastdarmes und deren Genese befasst, erheischt unser Fall viel weitere entwickelungsgeschichtliche Gesichtspunkte und vor allem ein Studium der Vorgänge in jener Zeit der Entwickelung, in der die Anlagen für Blase und Darm ihre völlige Trennung beginnen. - Es handelt sich ja hier um eine Ektopie der Blase, zu welcher sich eine Missbildung von Seiten des Enddarmes gesellt hat, so dass eine einheitliche Betrachtung dieser versehiedenen Organanlagen angehörigen Missbildung nöthig erscheint.

Bis vor Kurzem waren es für Entstehung der Spaltbildungen an Blase und Harnröhre zwei Ansichten, die am meisten Anklang fanden.

Einerseits glaubte man lediglich eine Hemmungsbildung, ein Stehenbleiben auf einer unvollendeten Entwickelungsstufe annehmen zu müssen, andererseits wurde die Ursache der Spaltbildungen in einer mechanischen Spannung der Harnwege durch den Urin gesucht.

Die Begründer und ersten Verfechter letzterer Anschauung waren Roose (55) und Credé (42). Sie suchten besonders die Endursache in einem mangelhaften Verschluss der vorderen knöchernen Beckenwand. Während Letzterer eine ungenügende Ver- 
474 r. Steinb üchel, Ueber Nabelschnurbruch u. Blasenbauchspalte

einigung der Beckenhälften als ursächlich beschuldigte, dachte Ersterer an eine durch ein Trauma erfolgte Sprengung der Symphyse. - Duncan (44), der ebenfalls ein Anhänger der Berstungstheorie war, glaubte eine Behinderung des Urinabflusses beschuldigen zu müssen. - Viele stimmten dieser Theorie bei, doch fanden sie auch viele Gegner, die sich für Annahme einer Hemmungsbildung aussprachen, wie vor Jahren Meckel (55) und viel später Ahlfeld (37), Winckel (68), Steiner (63) und Thiersch (66).

Diese in ihren Grundzügen geschilderten Lehren machten durch die Beurtheilung der jeweiligen Anhänger eine Reihe von Wandlungen durch, die sich jedoch meist auf Details beschränkten.

In letzter Zeit nahm Kaufmann (49) die durch Duncan (44) geförderte Berstungstheorie wieder auf, verlegte aber ihre Wirksamkeit in ein viel späteres Stadium, an das Ende des zweiten Monats, da zu dieser Zeit erst Urinsecretion stattfinde, somit vorher von Stauungsvorgängen nicht gesprochen werden könne. Es ist aber den Gegnern der Hemmungstheorie nicht gelungen, dieselbe $z u$ widerlegen und ist gegen dieselbe nur ins Feld zu führen, dass es niemals gelungen ist, Entwickelungsstadien anzutreffen, die den in Betracht kommenden Missbildungen entsprechen würden.

Für die Berstungstheorie wird als wichtigste Stütze das allerdings enorm seltene Vorkommen einer intrauterinen Verheilung der Blasenspalte angenommen. - Kaufmann (49) bemüht sich, die von ihm vertretene Hypothese bis zu den äussersten Consequenzen zu führen und zieht zur weiteren Festigung seiner Anschauungen Fälle von Dilatation der Urethra und der Ureteren herbei. -- Durch mehrere in neuester Zeit erschienene Arbeiten von Reichel (59 uud 60), der auf Grund eingehendster und sorgfältigster entwickelungsgeschichtlicher, eigener Studien das Studium der ganzen Frage nochmals von vorn anging, wurde die Lehre Kaufmann's in der entschiedensten Weise angegriffen und derselben auch ein grosser Theil ihres Bodens entzogen.

Die Anschauung Reichel's (l. e.) geht in Kurzem gefasst dahin, dass die grösste Mehrzahl der Missbildungen der Harnröhre und Harnblase, insbesondere die Spaltbildungen als Hemmungsbildungen aufgefasst werden müssen; ihre Entstehung ist an der Hand der entwickelungsgeschichtlichen Vorgänge zu verfolgen. - Reichel's Einwürfe gegen Kaufmann's Hypothesen gehen auch dahin, dass die Berstungstheorie 
bei der oft gleichzeitig vorkommenden Bauch-Blasen-Genital-Spalte jedwede Erklärung schuldig bleibt, ferner, dass bei den in Rede stehenden Spaltbildungen sehr häufig andere Missbildungen beobachtet wurden.

Der Einzige, der vor den bahnbrechenden Untersuchungen Reichel's seine Ansicht über die Entstehung der in Rede stehenden Missbildungen begründete, war Thiersch (l. c.); seine Erklärung aber, die auf einer fehlerhaften Zeitfolge des Beckenschlusses und der Cloakentheilung beruhte, konnte im besten Falle nur die Entstehung der Epispadie erklären.

Eine neue Wendung brachte die Entdeckung der doppelten Anlage der Allantois und spricht König (50) auf Grundlage von Untersuchungen und Beobachtungen von Bartels (40), Blasius (65), Neckel(l. c.) u. A. die Vermuthung aus, dass sich von innen her ein Hinderniss zwischen die symmetrischen Hälften der paarigen Organe dränge und ihre Vereinigung verhindere. Mit Ahlfeld(37) und Winckel(68) sucht er dieses Hinderniss in dem verlagerten Ductus omphalo-meseraïcus. Eine Stütze dafür suchte er darin, dass zuweilen eine gleichzeitige Missbildung vorkommt, „bei welcher der Darm und zwar das unterste Ende des Dünndarmes und der Blinddarm, falls er überhaupt vorhanden ist, in der Mitte der vorgefallenen Blase ausmündet, während der Mastdarm an seiner normalen Stelle fehlt." - Diese andeutungsweise hingeworfene Bemerkung von König (50) ist gerade für unsern Fall recht wichtig, indem von einer gleichzeitigen Ausmündung des Darmes, und zwar des Dünndarmes in die Blase gesprochen wird, also ein dem von uns erhobenen Befunde ungemein nahestehender. - Nach Reichel's Anschaung vermag auch diese Erklärung nicht.das Offenbleiben der einfachen oder doppelten Blase bei der Ectopie zu erklären, sondern würde höchstens die Doppelblasen verständlich machen. - In diese zum Theil recht erheblich von einander abweichenden Anschauungen konnte nur das genaueste Studium der Entwicklungsgeschichte des Schwanzendes Klarheit und Licht bringen, und es haben sich mit diesem Keibel (51), Strahl (64), Bonnet (39) und Vialleton (67) beschäftigt, ganz besonders aber Reichel(l. c.), der durch seine Untersuchungen, theils die Angaben der neueren Beobachter bestätigen, theils ergänzen konnte. Die für unsere Missbildungen in Frage kommenden Ergebnisse der Reichel'schen Untersuchungen sind folgende: „am frühesten entstehen die verschiedenen Arten der Bauchblasen- 
spalte und der Epispadie in Folge vollkommenen oder theilweisen Ausbleibens der Verschmelzung der Ränder der Primitivrinne zum Primitivstreifen in dem hinter der Aftermembran gelegenen Abschnitt. In die 4.--6. Woche des Fötallebens fällt die Entstehung der Cloakenmissbildung mit oder ohne gleichzeitige Blasenspalte, bedingt durch ausbleibende oder ungenügende Entwicklung und Verwachsung der Rathke'schen (62) Falten. Ihr geringster Grad ist die Persistenz des Cloakenganges.

Die mannigfachen Arten der Verschliessungen des Mastdarmes, wie die abnormen äusseren Ausmündungen desselben sind gleichfalls durch Verwachsung fötaler Gewebsabschnitte zu erklären.

Ein Zusammentreffen einer Blasenbauchspalte mit gleichzeitiger Cloakenbildung ist die hier in Rede stehende Missbildung.

Bei allen möglichen Formen dieser Missbildung führen die Harn- und Geschlechtswege sowie der Darm in eine gemeinsame Höhle, die entweder in normaler oder pathologischer Weise ausmündet. Die Ursache dieser Störungen ist in dem ausbleibenden oder ungenügenden Tiefertreten des Septum Douglasii gelegen. - Auf die entwickelungsgeschichtliche Bildung desselben müssen wir an dieser Stelle ganz kurz zurückkommen.

Als Cloake bezeichnen wir eine durch Umbiegung des Schwanzendes, die bauchwärts gerichtet ist, entstehende Höhle, welehe hinten den Darm, vorne oben die Allantois und zu beiden Seiten die Wolff'schen Gänge aufnimmt.

Die vordere Hälfte der Cloake ist mit niederem cubischen Epithel, die hintere mit höherem fast cylindrischen ausgekleidet, das in das Darmepithel übergeht. Zwischen beiden Hälften entstehen zwei wallartige Erhebungen, die Rathke'schen Falten und liegen ihre oberen Enden zwischen beiden Seiten der Einmündungsstelle des Darmes in die Cloake.

Die Rathke'schen Falten wachsen allmählig weiter nach abwärts und verschmelzen über der bis dahin offenen Darmrinne in der Medianlinie, wodurch diese in ein Rohr verwandelt wird. -

Durch die Verschmelzung dieser Falten und ihr Tiefertreten entsteht ein Septum, das obengenannte Septum Douglasii, und wenn es schliesslich ganz tief herabtritt, so ist die Folge eine gänzliche Abschliessung des Sinus urogenitalis vom Darme. - Die Genese 
der einzelnen Cloakenbildungen fällt in eine frühe Entwicklungsperiode, etwa in die 4.-6. Woche des Fötallebens, also etwas später als die Entstehung der Blasenspalte.

Jene Fälle, in denen die gemeinsame Oeffnung der Harn- und Geschlechtswege und des Darmes sich am unteren Ende der vorderen Bauchwand findet, sind eine Combination der Cloakenbildung mit gleichzeitig vorhandener Blasenbeckenbauchspalte. - Die Mündung des Darmes wurde bisher stets umpaar gefunden und zwar etwas höher oder tiefer gelegen als die Mündungen der Wolff'schen beziehungsweise Müller'schen Gänge. - In unserem Falle nun befinden sich die Oeffnungen des Darmes - dieselben sind zum Unterschiede von den bisher beschriebenen Fällen paarig vorhanden - oberhalb der Ureterenmündungen und der Ductus ejaculatorii. -

Wir kommen nun zu den beiden wichtigsten Punkten in der Erklärung und Deutung des von uns erhobenen Befundes:

1. Fast gänzliches Fehlen des Enddarmes und Einmündung des Dünndarmes (unteres Ende) in die Cloake.

2. Paarigbleiben oder - Werden der Darmöffnungen.

Für ersteres Verhältniss haben wir Folgendes anzuführen:

Fassen wir, bevor wir an einen Versuch der Beantwortung der beiden eben aufgestellten Fragen herangehen, kurz das Wesentliche unseren Fall entwickelungsgeschichtlich Betreffende zusammen, so können wir sagen: bis zur 5. Woche fehlen beim Embryo die äusseren Mündungen der Geschleehts- und Harnwege und des Darmcanales, der blind verlaufende Enddarm ist noch im Zusammenhange mit der Allantois. - Diese ist nach aussen hin offen und nimmt zugleich die Wolff'schen und Müller'schen Gänge auf; sie ist also die gemeinsame Oeffnung für alle diese Organe. Dieser Zustand kann andauern, indem bei Bauchspalte zugleich eine Blasenspalte besteht, während Darm- und Harncanal, sowie die Geschlechtswege in diese offene Blase münden. In anderen weniger hochgradig missbildeten Fällen haben wir eine Cloakenbildung mit Blasenspalte: die gespaltene Harnblase hat in der Mitte eine Oeffnung für den Darm, an den Seiten für die Ureteren und Ductus ejaculatorii; gewöhnlich findet sich die Mündung des Darmes "dem unteren Ende des Ileum entsprechend" (Birch-Hirschfeld(38)) oben und in der Mitte der Cloake, "das Colon fehlt oder ist durch einen blind endigenden Scblauch angedeutet." - Auch in unserem Falle fehlt fast der ganze Dickdarm 
478. v. Steinbüehel, Ueber Nabelschnurbruch u. Blasenbauchspalte

und ist nur eine relativ kleine blindsackartige Erweiterung am Ende des vorhandenen Dünndarmes als dem Dickdarm zugehörig anzusehen. Gerade so wie wir schon oben gesehen haben, dass die Blasenspalte enorm häufig von ganz anderen Missbildungen begleitet ist, und dass überhaupt verschiedene Formen der Missbildungen zu gleicher Zeit vorkommen, so muss man nicht nur die fehlerhafte Mündung des Darmrohres in die Blase als Hemmungsbildung auffassen, sondern ist wohl auch die Aplasie oder Hypoplasie des ganzen Dickdarmabsehnittes ungezwungen durch eine Hemmungsbildung zu erklären. Es wäre vielleicht denkbar, dass die Anlage zur Ausbildung des ganzen Dickdarmes vorhanden ist, dass dieselbe aber nach dem Zustandekommen einer höher oben gelegenen abnormen Communication mit der Körperoberfläche gleichsam als functionslos gewordenes Organ auf der niedrigen Entwickelungsstufe zur Zeit der Entstehung der Missbildung stehen bleibt, oder sogar vollständig verkümmert. -

Lntwickelungsgeschichtliche genaue Untersuchungen über die Entwicklungsverhältnisse des Dünndarmes und Dickdarmes, die sich mit den hier in Frage kommenden Dingen beschäftigen, sind bisher noch nicht gemacht, und Reichel (1. c.) selbst, der die Entwicklungsverhältnisse der Harnwege so eingehend studirt hat und zu vielfach ganz neuen und recht einleuchtenden Resultaten gekommen ist, hat sich mit der Genese des Darmrohres nicht befasst.

Aus den Angaben der diesbezüglichen Lehrbücher jedoch ist es nicht möglich, sich ein sicheres und überzengendes Urtheil über diese Dinge zu verschaffen. - In den hierher gehörigen Fällen wurde beobachtet, dass in den widernatürlichen After in der Regel noch ein kurzes, blindsackähnliches, abgeschlossenes Darmstück, das für Dickdarm gehalten werden musste, einmündet.

Dieses Darmstïck kann man sowohl auf partielles Persistiren der sonst verkümmerten Dickdarmanlage zurückführen, als auch auf eine im untersten Abschnitt der Cloake stattfindende Verschmelzung der Rathke'schen Falten (siehe oben), die von beiden Seiten her kommend sich vereinigen. - Es wäre demnach die Vereinigung im unteren Ende normal vor sich gegangen, an einer böher gelegenen Stelle aber, dem Orte der Bildung des widernatürlichen Afters entsprechend, dieselbe ausgeblieben. Um nun das Vorhandensein $\mathrm{z}$ weier Darmöffnungen in der Blase einem Verständniss etwas zugänglicher zu machen und eine Erklärung dieser merkwürdigen Verhältnisse zu versuchen, müssen wir 
für einen Augenblick von der Entwickelung der Allantois sprechen. - Durch die Untersuchungen Gasser's (47) wissen wir, dass die Allantois nicht im wahren Sinne des Wortes paarig angelegt ist und dass eine paarige Anlage dadurch vorgetäuscht wird, dass zu einer gewissen Zeit der Entwickelung die Wand symmetrisch zur Mittellinie in Form zweier Wülste vorspringt.

Jene wenigen in der Literatur bekannten Fälle von doppelter Blase ohne Blasenspalte und ohne Cloakenbildung waren leicht rerständlich bei der Annahme einer paarigen Anlage der Allantois und der Entwickelung der Harnblase aus ihr. - Reichel (l. c.) aber hält dafür, dass die mediane Scheidewand auf einer Verwachsung der Vorderwand der Blase mit einer von der Rückwand normaler Weise vorspringenden, in der Mittellinie gelegenen Leiste beruhe; diese Verlöthung soll zu einer Zeit geschehen, da die Blase noch mit dem. Darm communicirt. - Nach den Untersuchungen von Bartels, Blasius, Meckel (l. c.) und Scarenzio (65), die wir schon zum Theil oben wiedergegeben haben, hält König es für wahrscheinlich, "dass sich von innen ein Hinderniss zwischen die bilateral symmetrischen Hälften der noch nicht vereinigten Organe drängt und ihre Vereinigung hindert".

Wenn wir uns auf diesen allerdings mit den Untersuchungen Gasser's, Rèichel's und Anderer widersprechenden Standpunkt stellen würden, so wäre eine Erklärung der gedoppelten Ausmündung des Darmrohres in die Blase nicht allzu schwer. - Wir würden folgende Erklärung dafür als geltend oder wenigstens recht wahrscheinlich halten:

Der Ductus omphalo-meseraicus, der in Obliteration begriffen ist, oder bereits völlig verschlossen ist, drängt sich zwischen die beiden noch getrennten Anlagen der Harnblase (siehe oben Ahlfeld, Winckel, König) und verlöthet sich mit jeder der beiden Blasenanlagen. Durch den Druck des Meconiums, der im Ductus immer bdeutender wird, da ja der Dickdarm nicht ausgebildet ist und sich an das unterste flium nur ein kleiner, blind endigender Darmtheil (Coecum) anschliesst, erfolgt schliesslich in die vielleicht mittlerweile schon vereinigten Blasenhälften der Durchbruch, so dass wir symmetrisch von der Mittellinie zwei Oeffnungen, die in das Darmrohr führen, sehen. - Dadurch wäre es auch erklärt, dass gleich hinter den beiden, in die Blase führenden Oeffnungen das Darmrohr wieder einfach wird, wenn auch nur auf 
eine kurze Strecke, um dann wieder sich zu theilen in einen zuführenden, das untere Ilium darstellenden, auch mikroskopisch als Dünnidarm erwiesenen Schenkel, und in einen kleinen für Coecum anzusprechenden, blind endigenden Schenkel. -- Diese Erklärung hätte insofern manches für sich, als der in unserem Falle erhobene Befund einer ziemlich tiefen Furche in der Mitte des zu Tage liegenden Harnblasenstückes mit "narbenähnlichem" Aussehen den Gedanken an eine später erfolgte Vereinigung zweier ursprünglich getrennt angelegter Theile nahelegt.

Nach den Untersuchungen Li eberkühn's (54), Keibel's (51), Retterer's (61) und besonders denen Reichel's (1. c.) entsteht die Harnblase, mindestens in ihrem unteren Antheile, aus der vorderen Hälfte der Cloake selbst; die Allantois betheiligt sich an ihrer Bildung gar nicht, oder nur in recht geringem Maasse.

Durch das Abwärtswachsen der Rathke'schen Falten und ihre Vereinigung in der Mittellinie bilden sie aus der bis dahin offenen Darmrinne einen Canal, das Darmrohr, und grenzen das betreffende Darmstück dadurch von dem vorderen Hauptabschnitt der Cloake, also von der späteren Blase ab.

Die in der Mittellinie sich vereinigenden Rathke'schen Falten bilden verschmolzen das Septum Douglasii, nehmen nach jeder Richtung an Ausdehnung zu und nähern sich dort dem Boden der Cloake, so dass nur noch ein enger Gang, der Cloakengang, die Verbindung zwischen Mastdarm und Sinus urogenitalis herstellt. Der Ursprung der Rathke'schen Falten liegt zu beiden Seiten symmetrisch unmittelbar neben dem Darm und gehen sie direct in die Wand desselben über. - Es sind also diese Falten eng verknüpft mit dem Vorhandensein des Darmes und zwar jenes Theiles des Darmes, der in die Cloake einmündet.

Es frägt sich 1. „kann jeder in die Cloake einmündende Darmabschnitt durch das Vortretenlassen der Rathke'schen Falten und deren mediane Vereinigung zum Schluss der Darmrinne und Erzeugung des Darmrohres führen, oder muss dies ein bereits differenzirter Abschnitt des Darmeanales sein". - 2. "kann bei vollständigem oder theilweisem Mangel oder Verkümmerung des Dickdarmes ein höher gelegener Darmabschnitt functionell die Rolle des Fehlenden spielen und findet in diesem Falle die Differenzirung zwischen Darm und Sinus urogenitalis in der gleichen Weise statt, wie unter normalen Verhältnissen?" 
Nachdem diese beiden Fragen gegenwärtig wegen noch ausständiger Untersuchungen über die Entwickelung des Enddarmes nicht stricte beantwortet werden können, so bleibt nichts anderes übrig, als auf Grund der gegenwärtig vorhandenen Kenntnisse eine Erklärung zu versuchen:

Bleiben die Rath ke'schen Falten in ihrer Entwickelung zurück, verwachsen sie nur zum Theile oder gar nicht mit einander und
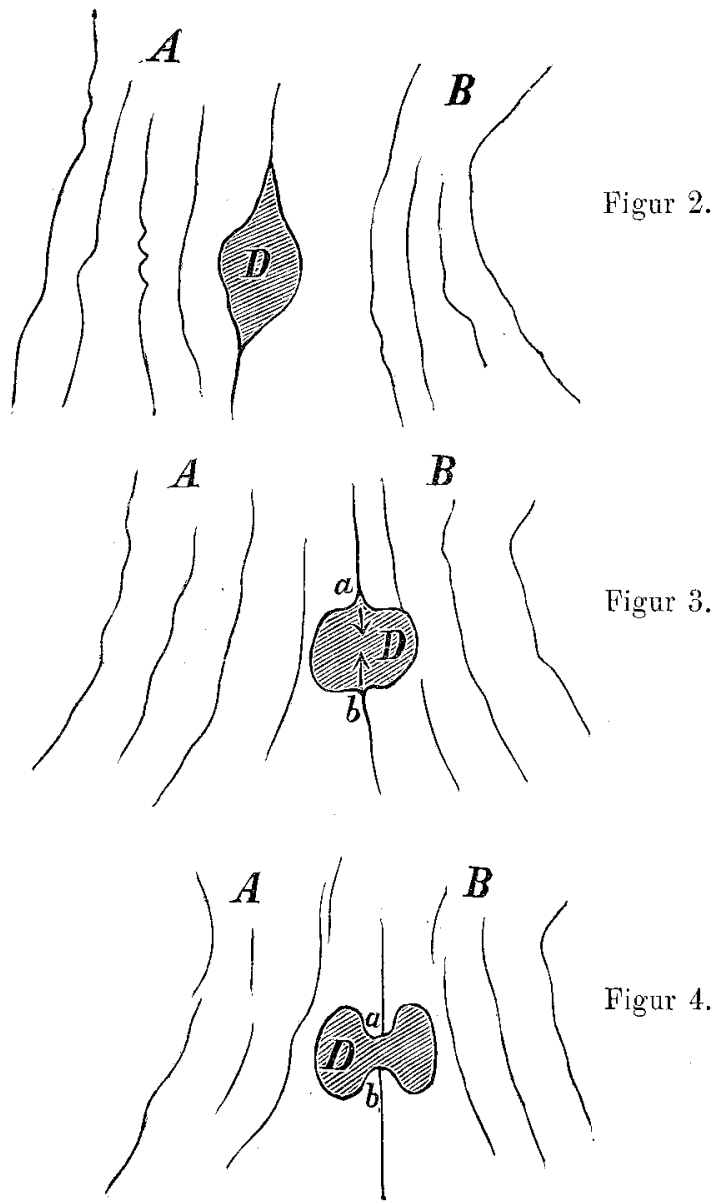

treten sie nicht so tief herab, dass sie das Cloakenseptum erreichen, so bleiben, wie es ja leicht zu verstehen ist, verschieden grosse Communicationen zwischen Blase und Darm bestehen.

Ein niedriger Grad dieser Missbildung ist das Bestehenbleiben des Cloakenganges; es ist die Harnblase zwar durch ein Septum 
Figur 5.

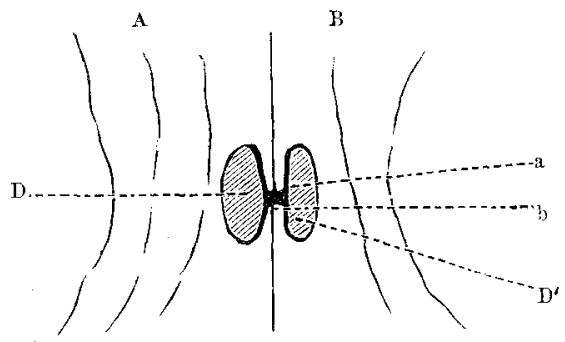

Frklärung der Figuren :

A B Rathke'sche Falten. - D Unpaare Mündung des Darmrohres. a b Sich entgegenwachsende median verschmolzene Theile der Rathke'schen Falten bilden bei Figur 5 den die unpare Oeffnung theilenden Sporn. - D D Parige Darmöffnung wie in dem rorbeschriebenen eigenen Falle.

rom Mastdarm geschieden, aber nicht vollständig geschlossen. Nun giebt es aber Fälle, in denen die Vereinigung der Rathkeschen Falten überhaupt oder höher oben ausbleibt; es schliesst sich die Darmrinne nicht zum Kanal; es ist eine Blasenspalte mit der Cloake combinirt; solche Missbildungen, welche den höchsten Grad der Becken-Bauch-Blasenspalte mit Cloakenbildung darstellen, kommen vor und ist von Barthels (40) über zehn derartigo Fälle berichtet worden.

Ganz unten mündet in solchen Fällen manchmal in den Anus praeternaturalis ein kurzes blindendigendes Stück Darm, das man entweder auf Erhaltenbleiben des sonst verlorengehenden, rom unteren Cloakenende sich fortsetzenden Enddarmes zurückführen kann, oder bei dem man denken kann, dass im untersten Abschnitte der Cloake eine Verschmelzung der Rathkeschen Falten stattgefunden habe und dieselbe nur in den oberen Theilen ausgeblieben sei.

Denkt man sich nun diese Verhältnisse sehr reducirt, so wird es gewiss einleuchten, dass durch das Ausbleiben der medianen Verschmelzung der Rathke'schen Falten an einer ganz beliebigen Stelle eine Communication zwischen Blase und Darm geschaffen werden kann. Dass dieser Darm nicht der Enddarm ist und ein solcher nur rudimentär in Form eines Blindsackes angehängt ist, hat mit dieser Communication absolut nichts zu schaffen.

Es ist nun ganz gut denkbar, dass, wenn die unpaare Oeff- 
nung "D" durch mangelhaften Verschluss von Seiten der Rathkeschen Falten "A und $B *$ bereits gebildet ist, es dennoch durch ein Entgegenwachsen in der Mitteliinie bei $r^{a}$ und $b^{*}$ (s. Fig. 2, 3,4 und 5) zu einer brückenartigen Verschmelzung kommen kann, die vielleicht in einer früheren Zeit des Fötallebens ziemlich mächtig ist, später aber durch den höher ansteigenden Druck des Meconiums im Darm stark gedehnt wird und somit nur mehr in Form einer ganz dünnen Gewebsbrücke die eigentlich unpaarige Oeffnung überspannt.

Bei genauer Erwägung der vielfache Variationen zeigenden Verschmelzungsverhältnisse der so wichtigen Rathke'schen Falten hat diese Annahme wohl nichts Unwahrscheinliches.

Bezüglich des früher erwähnten Punktes des Ausbleibens der Dickdarmanlage, wie des Verhältnisses der Rathke'schen Falten zu den einzelnen Abschnitten des Darmrohres, können wir nur abermals darauf hinweisen, dass den entwickelungsgeschichtlichen Untersuchungen Reichel's (l. c.) analoge Studien über die Entwickelung des Darmrohres, besonders dessen unteren Abschnitt nöthig wären, um diese noch recht dunklen Verhältnisse zu klären.

Figur 6.

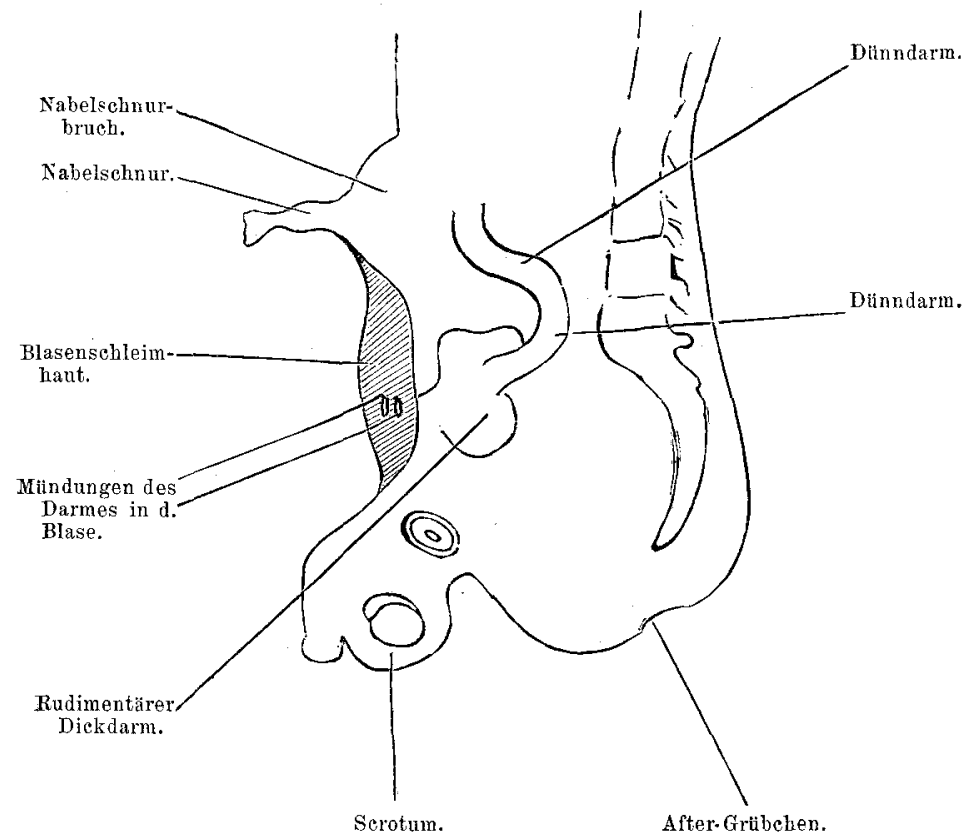

Schematische Abbildung unseres Falles. 
484 v. Steinbüchel, Ueber Nabelschnurbruch u. Blasenbauchspalte

Gerade in allerneuester Zeit sind einige Fälle veröffentlicht worden, die wenigstens zum Theil geeignet sind, in casuistischer und auch in genetischer Beziehung auf unseren so schwer zu deu-

Figur 7.

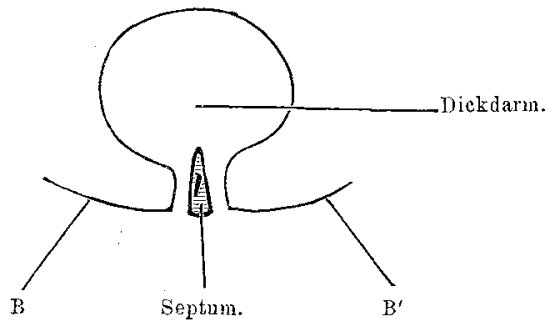

B n. B' Blasenschleimhaut.

Schematischer Durchschnitt durch Blase und Darm in der Höhe der Communicationsöffnungen bei unserem Falle.

tenden Fall ein, wenn auch etwas spärliches Licht zu werfen. M. Lange (53) berichtet über einen Fall von completer Verdopplung des Penis, combinirt mit rudimentärer Verdopplung der Harnblase und Atresia ani. -- Es handelte sich um ein 14 Tage altes Kind, bei dem ein vergeblicher Versuch gemacht wurde,

Figur 8.

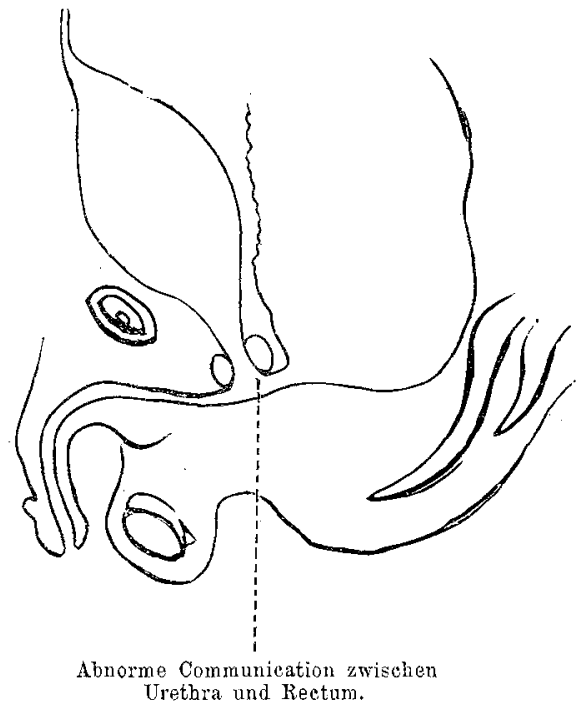

Schematische Abbildung des Falles Lange. 
vom After aus das untere blind geendigte Darmrohr aufzusuchen und dann ein Anus praeternaturalis suprainguinalis angelegt wurde. Das Kind starb an Erschöpfung. Der Befund war folgender (s. Fig. 8): es zeigten sich zwei vollständig gleichgeartete Penes in der Mittellinie aus der Radix Penis entspringend. Unter der Unterfläche jedes Penis ein deutlicher Hautkamm, der sich als Raphe auf den Hodensack fortsetzt. - Die inneren Organe des Kindes weisen nichts Abnormes auf. Auch Dünndarm und Dickdarm sind gehörig ausgebildet. Das S romanum ist kleinfingerdick, desgleichen das Rectum, das in der Höhe des II. Sacralwirbels blind endigt. - Nieren und Ureteren bieten keine Abnormitäten dar. - An der Harnblase finden sich ein Urachus und zwei Ligamenta lateralia. Die Blase ist auffallend gross, spindelförmig gestaltet. Sie ist durch ein medianes Septum in eine rechte und eine linke Hälfte getheilt; das Septum schliesst beide Hälften vollständig von einander ab. In jeder Blasenabtheilung mündete der entsprechende Ureter ein. Gerade neben dem unteren Ende des Septums liegt: jederseits das Orific. urethrae internum. Beide Urethrae communiciren mit einander nicht, dagegen findet sich eine feine Communication $z$ wischen rechter und linker Urethra und dem Rectum.

Wir haben es also hier wieder mit einem ähnlichen Falle zu thun, in dem eine abnorme Verbindung zwischen Blase und Darmcanal besteht; der Unterschied liegt nur darin, dass in unserem Falle sich zwei symmetrisch gelegene Oeffnungen direct in den untersten Abschnitt des vorhandenen Darmes einsenken, während hier die ebenfalls paarigen Verbindungsöffnungen nicht im Bereich der Blase, sondern im Bereiche der Urethra liegen und es sich hier um das Rectum handelt, während in unserem Falle ein solches gar nicht ausgebildet war, und wir es mit einem dem Coecum wahrscheinlich entsprechenden Darmstück $\mathrm{zu}$ thun hatten.

In dem Lange'schen Falle (Fig. 8) liegt diese Communication dicht unterhalb der breit ausgebildeten Prostata, welche beide Harnröhren umschliessend, zugleich ein Septum zwisehen beiden herstellt. Vergl. damit die schematischen Fig. 6 u. 7 .

Das Rectum endigt breit und blind oberhalb des Dammes. Die beiden Oeffnungen gegen die Urethra zu liegen rein lateralwärts.

Das ganze Knochengerüst ist der Norm entsprechend. Die 
486 v. Steinbüchel, Ueber Nabelschnurbruch u. Blasenbauchspalte

von Lange beschriebene Missbildung ist unstreitig sehr selten. Lange konnte in der ganzen hierhergehörigen Literatur nur drei hjerzu in engerer Beziehung stehende Fälle finden. Ein Fall ist beschrieben von Jenisch (48); in demselben handelte es sich um einen Knaben mit zwei nebeneinander befindlichen Penes. Beide waren perforirt und gelangte man durch sie in die Blase. An der hinteren Blasenwand befindet sich eine Oeffnung, die in das untere Dickdarmende mündete. Eine Analöffnung fehlte. Vergeblicher Versuch der Anlegung einer Analöffnung. Auch in diesem Falle handelte es sich um eine abnorme Verbindung zwischen den Harnwegen und dem Darmtract und war diesmal die Verbindung sogar im Bereiche der Harnblase vorhanden, nur handelte es sich dabei, soweit man die Angaben verfolgen kann, um eine unpare und nicht um eine symmetrische Verbindung zwischen beiden Organen. - Ein zweiter Fall, nur intra vitam behandelt, ist von Acton (36) und Velpeau beschrieben worden. -- Von einer gleichzeitigen Atresia ani neben der bestehenden Verdopplung der Penes ist nichts erwähnt; daher haben auch diese Fälle für uns nur ein untergeordnetes Interesse.

Der dritte Fall ist von Küttner (52) aus der Bruns'schen Klinik mitgetheilt und handelt es sich um ein Museumspräparat, bei dem sich verdoppeltes Scrotum mit je einem Testikel und doppeltem Penis finden. Blase sowie Anfangstheil der Urethra einfach; letztere theilt sich unterhalb der Prostata in zwei Röhren, von denen je eine zum betreffenden Penis verläuft. Gerade in der Prostata liegt die Mündung eines feinen Canals, der in das Rectum führt. Ob Atresia ani in diesem Falle vorhanden war oder nicht, ist wegen Detectes am Präparate nicht entscheidbar. Also auch in diesem Falle treffen wir eine uns sehr interessirende Missbildung, nämlich eine unpaare Verbindung zwischen Pars prostatica urethrae und Rectum.

Wenn man nun die drei vorhandenen etwas genauer geschilderten Fälle vergleicht, den Fall von Lange, den von Jenisch und endlich den von Kütt'ner, so muss bei ruhiger Ueberlegung folgendes auffallen. Drei Fälle von Doppelbildung des Penis; in einem Falle Septumbildung in der Blase: in allen drei Fällen Verbindungsgang zwischen Harnwegen und letztem verhandenen Darmstück. Es handelt sich also in allen drei Fällen um eine entwicklungsgeschichtlich nicht nur den Genitalhöcker, sondern auch 
den Sinus urogenitalis mitbetreffende Verdopplung der ursprünglichen Anlage.

Wir können nun in unserem Falle völlig von der Verdopplung im äusseren Genitale absehen und müssen aus den eben geschilderten Fällen nothwendigerweise den Schluss ziehen, dass eine Verdopplung im Sinus urogenitalis meist verbunden ist mit einer abnormen Communicationsöffnung zwischen Harnwegen und Darmcanal. - In dem Falle von Lange lässt sich die Communication zwischen Rectum und beiden Harnröhrenanfangstheilen vielleicht darauf zurückführen, dass entweder die Trennung der Cloake in einen vorderen und hinteren Abschnitt durch die lateral vorwachsenden Falten [Keibel (l. c.), Rathke (l. c.)] keine völlige geworden ist, oder aber es ist die Scheidewand so schwach angelegt, dass sie bei der später eintretenden Ueberfüllung des blind endenden Rectums barst und nunmehr die Verbindung mit den beiden Harnröhren eintrat.

Auch wir haben bei Erklärung unseres noch viel merkwürdigeren Befundes derartige Erklärungsversuche gemacht und verweisen wir auf das darüber an dem betreffenden Orte Gesagte.

Ein Septum, das die ganze Blase durchsetzt, ist bereits ausser dem Lange'schen Falle beobachtet und zwar von Meckel (Handbuch I. S. 652). Es liegt bei diesem Befunde wohl die Annahme nahe, dass es sich um eine rudimentäre Verdopplung der Allantois, also der Matrix in ihrem oberen und mittleren Theile handelt; in ihrem unteren Theile gebt ja die Harnblase, wie wir bereits an anderer Stelle nach neueren Arbeiten erwähnt hatten, aus dem Sinus urogenitalis hervor.

Es liegt nun die Frage vor, ob nicht in frühen entwicklungsgeschichtlichen Stadien Verdoppelungen der Harnblase eingeleitet sein und bei Entstehung von Missbildungen zu abnormen Oeffnungen fübren können, dass später aber im weiteren Verlaufe der Entwicklung es nicht zur Bildung eines gedoppelten Organs kommt, sondern das Septum rudimentär bleibt und nur vielleicht an der Hinterwand der vorne offenen Harnblase eine kammartige Erhebung als Rest der versuchten Verdopplung zu sehen ist.

In letzterem Falle ist die Annnahme einer frühzeitigen Doppelverschmelzung mit dem unteren Ende des Darmrohres ja naheliegend und wäre dadurch die paarige Blasenöffnung gegen den Darm leichter verständlich. - Ein anderer uns interessirender Fall, 
der sich auch entwickelungsgeschichtlich eingehender vertieft, ist von Dienst (43) aus dem pathologischen Institut von Breslau veröffentlicht.

In diesem Fall handelt es sich um ein drei Tage altes Kind mit meteoristisch aufgeschwollenem Bauche. Seit der Geburt war Stuhl nicht abgegangen. Vom Perinaeum aus liess sich ein Darm nicht finden und es wurde daher zur Anlegung eines Anus praeternaturalis in inguine geschritten. In der freien Bauchhöhle Luft und facculent riechendes Exsudat. Ein als Darmschlinge angesehenes Gebilde wird mit den Bauchdecken vernäht. Nach seiner Eröffnung ergicsst sich in reichlicher Nenge helle urinähnliche Flüssigkeit. Erst später wird der mit Meconium äusserst stark gefüllte Dickdarm gefunden und an demselben der widernatürliche After angelegt. Am Abend desselben Tages starb das Kind.

Section: Die Bauchhöhle war mit wenigstens $200 \mathrm{ccm}$ einer grünlichen, mit Flocken vermischten Flüssigkeit erfüllt, Erscheinungen einer Allgemeinperitonitis. Die linke Niere erweist sich als ein vergrössertes, schwappendes Organ, der dazu gehörige Ureter kleinfingerdick, die rechte Niere ist an der Oberfläche gelappt, der Vreter annähernd normal (jedenfalls etwas verdickt). Die Harnblase ist in einen sehr grossen, im kleinen Beeken und hinter der Symphyse liegenden Tumor verwandelt, ihre Wandung ist am Fundus ungefähr $9 \mathrm{~mm}$, am Grunde etwa $5 \mathrm{~mm}$ dick. Die Schleimhaut hyperämisch, am Fundus gefaltet, die Pars prostatica ist in das Cavum der Blase hineingezogen. Der Darm, an dem der widernatürliche After angelegt worden war, zeigt sehr ungleiche Weite, der am meisten erweiterte Theil, der circa $10 \mathrm{~cm}$ Umfang hat, und dem Rectum angehört, liegt im klemen Becken, in einer nischenartigen Vertiefung der hinteren Harnblasenwandung. Von da aus verengert sich dieser so stark ausgedehnte Darmantheil conisch. Die Blase hat nach hinten zu 2 Ausbuchtungen und zwischen diesen beiden liegt der sich verjüngende Theil dieses Darmes. Zwischen Kreuzbein und Blasengrund liegt dieser letzte sich verengende Theil und erstreckt sich von hier als feiner, nur noch $1 \mathrm{~mm}$ im Durchmesser haltender Gang noch $2 \mathrm{~cm}$ weit nach vorne, nach der Gegend der Prostata, wo er bindegewebig verschlossen in der Gegend des Colliculus seminalis unter dem Blasengrunde sein Ende erreicht.

Durch diesen Befund wird der von Dienst (1. c.) geschilderte Fall auch für uns von besonderem Werth.

Es handelt sich nunmehr abermals um eine, wenn auch nicht vollständig ausgebildete Communication zwischen unterem Ende des vorhandenen Darmcanals und den Harnwegen; nur endete dieser Canal, den wir in den letzten beiden Fällen als vollständig ausgebildete Communicationsöffnung angetroffen haben, in diesem Falle am Blasengrunde blind. 
Dienst behandelt nun zugleich ausführlich die Entstehung derartiger Missbildungen und betont vor allem das ontogenetische Verhalten des Darmantheiles und Urogenitalapparates.

Er bemerkt sehr richtig, dass in allen bisher beschriebenen derartigen Fällen Erklärungen auf entwicklungsgeschichtlicher Basis gesucht worden sind, aber die Anschauungen in jüngster Zeit haben sich gerade auf entwicklungsgeschichtlichem Boden so sehr verschoben, dass sie sich mit noch vor kurzem geltenden nicht mehr decken.

Seirie Ausführungen gründet er vornehmlich auf Arbeiten ron P. Reichel, Born, Schultze und Keibel (1. c.), Autoren, die wir schon vorher bei Erklärung unseres Falles kennen gelernt hatten und deren Untersuchungen wir in kurzem, soweit sie für denselben in Betracht kamen, wiedergegeben haben.

Dienst fasst aut Grund der vorliegenden Arbeiten seine Anschauungen über das Zustandekommen der verschiedenen Formen der Atresia ani auf dreifache Weise zusammen.

Diese Missbildungen verdanken ihre Entstehung einer reinen Bildungshemmung, einer abnormen Verwachsung fötaler Gewebsspalten, und endlich einer Combination dieser beiden Momente.

Die uns speciell interessirenden Missbildungen, besonders die abnormen Verbindungen zwischen Enddarm und Harnwegen, fasst er als reine Bildungshemmung im Bereiche der entodermalen Cloakenanlage auf, wie wir es auch früher schon gethan hatten. Als wesentlich ist noch anzuführen, dass gerade die Ausführungen von Dienst sich hauptsächlich mit der Atresia. ani als Bildungshemmung besehäftigen und die sogenannten „äusseren" Fistelbildungen, wie die Atresia ani perinealis scrotalis, suburethralis etc. eingehender behandelt werden, während die uns interessirenden Formen früher entwicklungsgeschichtlich schwer zu deutender Vorgänge weniger Berücksichtigung fanden.

Wenn die Rathke'schen Falten sich gar nicht oder nur ganz rudimentär bilden und dem entsprechend keine Trennung der ursprünglichen Cloake bewirken können, dann münden Mastdarm, Harnblase, Wolff'sche und Müller'sche Gänge in einen und denselben Hohlraum und bestehen ungefähr dieselben Verhältnisse, wie bei einem menschlichen Embryo von 4-6 Wochen. Demnach lassen sich Fälle von wirklicher Cloakenbildung als einfache Bildungshemmungen aussprechen. 
490 r. Steinbüchel, Ueber Nabelschnurbruch u. Blasenbanchspalte

In einem von Voll (69) erwähnten Falle war bei einem $40 \mathrm{~cm}$ langen männlichen Foetus keine Spur von Anal- noch UrethralOeffnung vorhanden. Der Penis war rudimentär. Blase und Mastdarm standen durch einen engen Gang in Verbindung. Dieser Fall gehört in die Reihe der soeben erwähnten Missbildungen.

Es ist ferner möglich, dass die Rathke'schen Falten zwar in der Anlage ganz regelrecht gebildet sind, dass aber die Versehmelzung zum Septum Douglasii nur theilweise und unvollkommen geschieht. Es kann auch vorkommen, dass sie sich median vereinigen, aber nicht tief genug herabtreten. Durch diese Abnormitäten in der Verwachsung der Rathke'schen Falten lassen sich wohl unschwer alle hierher gehörigen Missbildungen erklären, wie wir es ja auch schon oben gethan hatten.

Als ein geringster Grad von Missbildung würde das Bestehenbleiben des sogenannten Reichel'schen Cloakenganges zu gelten haben.

Wie wir schon mehrmals betont haben, fällt die Zeit der Entwicklung der Missbildung in die 4.-6. Woche des Foetallebens.

Hier näher auf die entwicklungsgeschichtliche Frage der Atresia ani einzugehen, erscheint aus dem Grunde nicht angezeigt, weil es sich ja in unserem Falle, obwohl eine normale Analöffnung vollständig gefehlt hat, trotzdem eigentlich nicht um eine Atresia ani, sondern um eine mit einer Atresia ani et recti verbundene Hypoplasie, beziehungsweise Aplasie fast des ganzen Dickdarmes gehandelt hat.

Es sind also vielmehr jene Fälle für uns maassgebend, in denen über gleichseitige Missbildungen bei Atresia ani berichtet wird.

So findet man bei Dicnst eine Arbeit von Brisken (41) erwähnt, die von einer "mangelhaften Ausbildung anderer Theile des Darmkanales spricht, ferner Blasenspalte, Hermaphroditismus und ähnliche Missbildungen hervorhebt." In einem Falle von Pretty (56) bestand die Analöffnung eines Knochen nur in Form einer flachen Einstülpung, während das Colon ascendens als Blindsack an Promontorinm endete. Also auch dies ist ein Fall, in dem eine Hypoplasie eines Theiles des Darmrohres, des Dickdarmes, zur Beobachtung kam.

Eine ebenfalls in neuerer Zeit erschienene Arbeit von Rasch (57) 
beschäftigt sich mit der weiblichen Epispadie und fissura vesicae inferior, hat aber für die Erklärung unseres Falles keine weitere Bedeutung.

Es handelt sich bei dem ron uns beschriebenen Falle jedenfalls um eine sehr seltene Missbildung.

Sie gehört in die Reibe jener Missbildungen, bei denen abnorme Mündungen des atretisch verschlossenen Darmes in andere Organe gefunden werden; die dabei auftretenden paarigen Oeffnungen sind durch Abnormitäten der Verschmelzung der Rathke'schen Falten zu erklären; es ist dabei aber auch zu bedenken, dass die Harnblase aus zwei ganz verschiedenen Abschnitten entsteht, wenigstens nach der Anschauung von Born (1. c.), der glaubt, dass der Scheitel der Blase aus der Atlantois hervorgeht, wäbrend der ganze übrige Theil aus dem ventralen Theil der entodermalen Cloake sich entwickelt.

Das, was am meisten in unserem Falle zum Nachdenken anregt, ist das unvollständige Vorhandensein des Darmes, der sich aber zur Harnblase doch so verhalten hat, als wie ein ausgebildeter und mit derselben analog dem Reichel'schen Cloakengang dureh zwei Oeffnungen verbunden ist.

Das Verständniss derartiger Missbildungen und die schwierigen dabei in Betracht kommenden entwicklungsgeschichtlichen Vorgänge und Störungen kann nur durch eingehende und sorgfältige entwicklungsgeschichtliche Studien über die normalen Vorgänge in den in Frage kommenden Theilen und eine sorgfältige Sammlung und Aufzeichnung aller bisher beobachteten Falle von Missbildungen gefördert werden.

Wenn diese beiden Forschungswege Hand in Hand gehen und sich gegenseitig ergänzen, so werden die Schatten, welche noch vielfach in diesem Forschungsgebiete liegen, durch Licht ersetzt werden. Zweek der vorliegenden Mittheilung war es, einen Beitrag zu dem zweiten angedeuteten Wege der Erkenntniss zu liefern.

Graz, im November 1899. 
294 T. Steinbüchel, Ueber Nabelschnurbruch п. Blasenbauchspalte

\section{Einschlägìge Literatur,}

welche übrigens keinen Anspruch auf Vollständigkeit macht.

1) Ahlfeld, Dieses Archiv. Bd. 5.

2) Derselbe, Missbildungen. II.

3) Nrndt, Nabelschnurbruch mit Herzhernie. Centralbl. f. Gyn. 1896. No. 24.

4) Benedilit, Centralbl. f. Gyn. 1892. No. 40.

5) Both, Zeitschr. f. Geb. u. Gyn. Bd. I.

6) Berger, Traité de Chir. Bd. VI.

7) Breus, Zur Therapie der Hernia funicul. umbilic. Wiener ned. Wochenschrift. 1881.

8) Derselbe, Ueber Behandlung des Nabelschnurbruches. Samml. klin. Vortr. n. F. Leipzig. No. 77. Gyn. No. 30.

9) Condamin, Arch. prov. de chir. I.

10) Colla, Centralbl. f. Gyn. 1890. S. 380.

11) Caldwell, CentralbI. f. Gyn. 1887. No. 2.

12) Cruveilhier, Anatomie pathologique du corp. humain. Il. tome, livrais 31. 1835-1842. - Derselbe, Wiener med. Wochenschr. 1881. No. 41.

13) Dúnlap, Centralbl. f. Gyn. 1888. No. 43.

14) Eckerlein, Zeitschr. f. Geb. u. Gyn. 15. Bd. 1888. „Ein Fall erfolgreich oper. Nabelschnurhernie."

15) Fleischmann, Prag. med. Wochensch. 1882. No. 28. Casuist. Mitth. aus d. II. geb. Kl.

16) Derselbe, Centralbl. f. Gyn. 1887.

17) Felsenreich, Wiener med. Presse. 1883. Zur Therap. A. Hern. funic. umbil.

18) Goodlee, Med. Times and Gazette. 1883.

19) Hecht, Fall von Nabelschnurbruch. Münchener med. Wochenschrift. Bd. XLI. S. 1037.

20) Kocher, Hernien im Kindesalter. Gerhardt's Handbuch der Kinderkr. 6. Bd. 1880.

21) Krukenberg, Dieses Archiv. 20. Bd. 1889. Zur Therapie d. Hern. funic. umbil.

22) Karewsky, Die chirurg. Krankheiten des Kindesalters. 1894.

23) Lindfors, Zur Lehre vom Nabelschnurbruch und seiner Behandlang, mit besonderer Berücksichtigung der Fortschritte des letzten Decenniums. 1882-1891. Samml. klin. Vortr. No. 63. Gynäk. No. 26. S. 617 bis 636 (mit reicher Literatur).

24) Derselbe, Centralbl. f. Gyn. 1884. S. 472.

25) Derselbe, Centralbl. f. Gyn. 1889. S. 482.

26) Macdonald, Frommel's Jahresber. über d. Fortschr. in Geb. und Gyn. $4 \mathrm{Bd}, 1891$.

27) Olshausen, Zur Therapie der Nabelschnurhernien. Dieses Archiv. 29. Bd. 1887 . 
28) Piperno, Centralbl. f. Gyn. 1888. No. 28.

29) Poppelmann, Die Behandlung des Nabelschnurbruches. Marburg 1894.

30) Piering, Prag. med. Wochenschr. 1896. No. 31. S. 342.

31) Runge, Dieses Archiv. 41. Bd. 1891. Mittheilung aus der Göttinger Frauenlilinik. - Erfolgreishe Operation eines Nabelschnurbruches durch Laparotomie.

32) Reuter, Centralbl. f. Gyn. 1887. No. 10.

33) Stolpinsky, Centralbl. f. Gyn. 1889. No. 11.

34) Trewes, Lancet 1884.

35) Thudichum, Illustr. med. Zeitung. 1852.

36) Acton, Medico-chirurg. Transactions. London 1896. pag. 103.

37) Ahlfeld, Dieses Archiv. X u. XI.

38) Birch-Hirschfeld, Handb. d. pathol. Anatomie. 1896. I. Bd.

39) Bonnet, Ueber die Entwickelung der Allantois und die Bildung des Afters bei den Wiederkäuern etc. Anat. Anz. 1888.

40) Bartels, M., Ueber die Bauchblasengenitalspalte, ein bestimmter Grad der sogen. Inversion der Harnblase. 1867. Dissert.

41) Brisken, I. Diss. über Atresia ani und ihre Behandlung. Bonn 1888.

42) Credé, Die Krankheiten des weiblichen Beckens. 1793. Berlin.

43) Dienst, Ueber Atresia ani congenita nebst Mittheilung eines Falles von Atresia ani urethralis ete. Virchow's Archiv. Bd. 154. Heft 1.

44) Duncan, Edinburg. med. Journ. 1865.

45) Eppinger, Atresia ani. Prager med. Wochenschr. 1880.

46) Frank, R., Ueber die angeborene Verschliessung des Mảstdarmes mit den begleitenden inneren und äusseren Fistelbildungen. Wien 1892.

47) Gasser, Beiträge zur Entwickelungsgeschichte der Allantois, der Müllerschen Gänge und des Afters. 1874.

48) Jenisch, Württemberg. Correspondenzbl. 1840.

49) Kaufmann, Verletzungen und Krankheiten der männl. Harnröhre.

50) König, F., Lehrbuch der spec. Chirurgie. VI. Aufl.

51) Keibel, F., Die Entwickelungsvorgänge am hinteren Ende des Meerschweinchenembryos. Arch. f. Anatomie u. Physiologie. 1888. Anatom. Abhandl.

52) Küttner, Ueber angeborene Verdoppelung des Penis. Beitr. zur klin. Chirurgie. Bd. XV. Heft 2.

53) Lange, Ueber complete Verdoppelung des Penis, combinirt mit rudimentärer Verdoppelung der Harnblase und Atresia ani. Ziegler's Beitr. Bd. XVIII. pag. 223.

54) Li eberkühn, Marburger Sitzungsberichte.

55) Meckel, J. F., Handbuch der pathol. Anatomie. Bd. I.

56) Pretty, Med. Gazette. Vol. XLVII.

57) Rasch, Beitr. zur klin. Chirurgie. Bd. XVIIr. Heft 3.

58) Roose, De vesicae urinariae prolapsu. Göttingen 1793.

59) A. v. Rosthorn, Unvollkommene Cloakcubildung (Fistula rectovestibularis) bei gleichzeitiger normaler Ausmündung des Darmes. Wiener klin. Wochenschr. 1890. No. 10. 
494 v. Steinbüchel, Ueber Nabelschnurbruch u. Blasenbauchspalte etc.

60) Reichel, P., Die Entstehung der Missbildungen der Harnblase und Harnröhre. Archiv f. klin. Chir. Bd. 46.

61) Derselbe, Die Entwickelung des Dammes und ihre Bedeutung für die Entstehung gewisser Missbildungen. Zeitschrift f. Geb. und Gynäli. Bd. XIV.

62) Retterer, Sur l'origine et l'évolution de la reg. ano-génit. des mammif. Journ. de l'anat. et de la phys. 1890.

63) Rathke, Beobachtungen und Betrachtungen über die Entwickelung der Geschlechtswerkzenge bei den Wirbelthieren. Neue Schriften der Naturforsch. Gesellschaft in Danzig. Bd. I. 1825.

64) Steiner, Archiv f. klin. Chirurgie. Bd. XV.

65) Strahl, Zur Bildung der Cloake des Kaninchenembryo. Archiv f. Anat. und Phys. 1886. Anat. Abth.

66) Scarenzio, cit. bei König.

Blasius, cit. bei König. Lehrbuch der spec. Chirurgie. 6. Auf. 1894. II. Bd.

67) Schwarz, C., Ueber abnorme Ausmündungen der Ureteren und deren chirurg. Behandl. (nebst Bemerkungen über die doppelte Harnblase).

68) Thiersch, Archiv f. Heilkunde. Jahrg. X.

69) Vialleton, Essai embryologique sur le mode de formation de l'exstrophie de la vesice. Arch. provinc. de chir. 1892. No. 3.

70) Winckel, Die Krankheiten der weibl. Harnröhre und Blase. 1885. 